\title{
Integrative strategy for improving cancer immunotherapy
}

\author{
$\mathrm{Xuetao}^{\mathrm{Cao}}{ }^{1}$
}

Published online: 26 April 2016

(C) Springer-Verlag Berlin Heidelberg 2016

Cancer immunotherapy has decisively advanced to the forefront of cancer treatment as a consequence of the impressive clinical responses of immune checkpoint blockade and adoptive $\mathrm{T}$ cell therapy. However, the current immunotherapeutic approaches have been shown to benefit only a minority of cancer patients. How to improve the efficacy of immunotherapy as an important modality for the treatment of advanced cancer attracts much attention now. Addressing this challenge requires a better understanding of molecular and cellular mechanisms for tumor escape of immunological control and identification of more effective ways in eliminating tumor cells, which will enable us to translate the basic knowledge of tumor immunology to the development of new immunotherapeutic strategies effective in clinical practice. In this issue of the Journal of Molecular Medicine, we are pleased to present five review articles that highlight recent advances in our understanding of basic aspects of tumor immunology and summarize current developments in new cancer immunotherapeutic strategies.

Considering that only a fraction of cancer patients with certain types can achieve the durable clinical benefit from the immune checkpoint blockade, in recent years, the combination strategies of cancer immunotherapy together with conventional chemotherapy or radiotherapy have been encouraged and extensively investigated both in preclinical and

Xuetao Cao

caoxt@immunol.org

1 National Key Laboratory of Medical Molecular Biology and Department of Immunology, Institute of Basic Medical Sciences, Chinese Academy of Medical Sciences, 5 Dongdan Santiao Road, Beijing 100005, China clinical studies. This relies on the conceptual advance in the characterization of immunogenic cell death (ICD) of cancer cells induced by these traditional approaches, and polarization of regulatory immune cells and inflammatory stromal cells in tumor microenvironment, from tumor-promoting to tumorinhibitory status, by chemotherapy and radiotherapy if used appropriately at timing, dosage, frequency, fractionation, and treatment sequences. It is usually accepted that the conventional chemotherapy and radiotherapy can mediate direct killing of tumor cells but can also damage the immune system of the patients at the same time. However, more and more evidence indicates that chemotherapy and radiotherapy can induce antitumor immune response by promoting ICD in cancer cells and subverting the immunosuppressive tumor microenvironment. In addition, both innate and adaptive immune systems of the host contribute critically to the outcomes of chemotherapy and radiotherapy in the treatment of cancer. For example, Fu's lab recently demonstrated that type I IFN is required for the control of cancer by local radiotherapy, and also showed that the combination of radiotherapy with antiPD-L1 antibody therapy exhibits synergistic effects in the treatment of tumor models by enhancing effector functions of cytotoxic T cells (CTLs) and inhibiting the accumulation of immunosuppressive myeloid-derived suppressor cells (MDSCs) [1]. It is well known that chemotherapy can initiate innate responses by inducing the cancer cell to release alarming substance and innate factors to activate antigenpresenting cell function. Furthermore, chemotherapeutic drugs have been shown to regulate tumor cell immunogenicity and achieve therapeutic benefits by activating the adaptive immune system. For example, the combination of cisplatin and paclitaxel at low dosage induces a strong tumor-specific $\mathrm{CD} 8+\mathrm{T}$ cell response in both mice and patients. Combined use of 5-FU with cisplatin increases both $\mathrm{CD} 4+$ and CD8+ T lymphocytes in the tumor microenvironment in cancer 
patients. Combined use of immunogenic drugs oxaliplatin and cyclophosphamide can initiate host antitumor T cell immunity in some cancer patients and successfully sensitize these patients to checkpoint blockade [2]. Therefore, it is important to understand the immunological mechanisms and rationales of the strategies for effectively combining conventional treatment with immunotherapy to improve the clinical outcome for cancer patients. In their review article, $\mathrm{Fu}$ and colleagues provide the mechanistic insights into the increased antitumor effect of the combination therapy and summarize the advances in both experimental and clinical studies on the therapeutic effect of the combining strategy [3].

Tumor-host interaction has always been a hot topic in this field. Now, we have accumulated extensive data about interaction of tumor cells and non-tumor cells including stromal cells and immune cells. Many factors, both intrinsic and extrinsic, can affect the interactions, promoting or inhibiting tumor growth and metastasis or even regulating the sensitivity of tumor cells to conventional therapies. Microbial communities inhabiting the human intestine have become important environmental factors involved in many health- and disease-related processes including inflammatory diseases and cancer. More recently, the gut microbial flora has unexpectedly shown to be able to regulate tumor microenvironment and can determine the outcome of the cancer immunotherapy via immune checkpoint blockade [4, 5]. Ma, Zitvogel, and colleagues now discuss the effects of certain cancer therapies on the changes of tumor microenvironment and highlight the importance of cellular stress responses especially microbiota-derived factors in the induction of immune response against cancer [6].

Rational design of new immunotherapeutic approach or combination strategies depends on the understanding of the balanced network of activation of antitumor immunity and tumor-induced immunosuppression. Tumors develop multiple means to actively help tumor cell escape of immunological control. Extraordinary efforts have been made to target tumor-induced immunosuppression by eliminating the regulatory cells and blocking the production of immunosuppressive factors. Through these joint efforts, the strength and/or duration of antitumor immune responses could be induced, and the long-term effectiveness of immunotherapies could be achieved. In the last decade, many kinds of regulatory cells have been identified in the immediate tumor microenvironment, the circulating system, and distant organs [7]. It is demonstrated that the increased number of regulatory cells and decreased expression of effector molecules with potent antitumor effects will attenuate the immune response against cancer and also promote tumor metastasis. For example, hepatic expression of the innate RNA sensor retinoic acidinducible gene I (RIG-I) contributes to the antitumor effect of type I interferon (IFN) therapy, and lower expression of RIG-I will lead to poor prognosis and reduce the antitumor effect of type I IFN therapy in hepatocellular carcinoma patients [8]. In this issue, Liu and Cao focus on the types of immunosuppressive cells and their mechanisms in promoting tumor immune escape and metastasis, and also outline some future directions in the development of cancer immunotherapy [9].

Inflammatory milieu consisting of regulatory cells and inflammatory cytokines contributes to tumor growth and metastasis. IL-6, IL-1 $\beta$, and TNF $\alpha$ are well known for their pathogenic inflammatory roles in the formation of malignancy. In recent years, one group of immune cells, innate lymphoid cells (ILCs), attracts much attention given their multiple functions in health and disease. ILCs that provide an early source of cytokines play an important role in homeostasis and inflammation, and the discovery of a diverse array of innate lymphoid cells with different cytokine expression profiles and migration patterns has provided insight into the molecular mechanisms that initiate and manipulate the inflammatory immune response physiologically and pathologically. Different cytokines derived from ILC subsets have been found to be involved in the carcinogenesis, tumor growth, and metastasis through interactions with other types of immune or nonimmune cells. IL-22, released from ILC3s and Th22, is elevated in the blood of many types of cancer hosts and also increased in the tumor microenvironment. Persistently elevated levels of IL-22 are associated with chronic inflammation, and development and progression of several types of cancer. Zou and colleagues have been investigating immunosuppression and tumor escape in the tumor microenvironment, and one of their studies showed that IL-22 can promote colorectal cancer cell invasiveness and stemness [10]. In this issue, Zou, Frankel, and colleagues summarize the cellular sources and targets of IL-22, and focus on the effects of IL-22 on different tissue environments, particularly in the context of cancer [11]. IL-22/IL22R signaling cascade can enhance cellular proliferation and invasion of multiple tissue types of cancer cells, contributing to both development of pre-neoplastic and maintenance of established tumors. This may provide mechanistic explanations for the observations that ablation of ILCs in a murine model of inflammation-mediated colon cancer results in abrogation of tumor formation, and also administration of anti-IL-22 antibody could ameliorate colitis symptoms and reduce tumor burden in the mouse model. Going forward, crosstalk of the IL-22/IL22R cascade with other inflammatory cytokines and inflammatory tumor stromal milieu, and more direct evidence definitively supporting the existence and functional role of local IL-22 in inflammation-induced tumorigenesis warrants further investigations.

IL-33, a member of the IL-1 family, has been shown to play critical roles in bridging innate and adaptive immunity through binding to its receptor ST2 (homolog of sulfotransferase 2). 
Since ST2 is widely expressed in many types of cells including ILC2s and T cells such as regulatory $\mathrm{T}$ cells (Treg), IL-33 can exert its multiple functions in T cell regulation, inflammation, and tumorigenesis, depending on the cell resources of IL-33 and the types of ST2positive targets. IL-33 is also found to be expressed in the tumor microenvironment and increased in the peripheral blood of patients with a variety of cancers. Many reports have suggested the involvement of IL-33 in tumor-associated inflammation and local immunosuppression. For example, tumor stromal expression of IL-33 contributes to tumor progression and metastasis by mobilizing MDSCs to tumor microenvironment and maintaining the immunosuppressive function of MDSCs within the tumor microenvironment. In a comprehensive review article by $\mathrm{Lu}$, Wang, and colleagues, the molecular characteristics of IL-33, the effects of IL-33 on the effector T cells, ILCs and myeloid cells during the homeostasis, cellular immunity and inflammation, the factors determining the tumor-promoting or tumor-inhibitory roles of IL-33, and how to selectively regulate IL-33 expression in tumor cells or tumor stroma to benefit immunotherapy of cancer have been summarized [12].

Taken together, these five review articles highlight important molecular and cellular mechanisms of tumorinduced immunosuppression and potential new targets of intervention, illustrate how to design new approaches of cancer immunotherapy to revert immunosuppression, and discuss the combination strategies of immunotherapy with conventional therapy. These reviews will undoubtly broaden our current understanding of the tumor immunology and immunosurvailence and inspire future innovations in cancer immunotherapy.

\section{References}

1. Tang H, Wang Y, Chlewicki LK, Zhang Y, Guo J, Liang W, Wang J, Wang X, Fu YX (2016) Facilitating T cell infiltration in tumor microenvironment overcomes resistance to PD-L1 blockade. Cancer Cell 29:285-296

2. Pfirschke C, Engblom C, Rickelt S, Cortez-Retamozo V, Garris C, Pucci F, Yamazaki T, Poirier-Colame V, Newton A, Redouane Y et al (2016) Immunogenic chemotherapy sensitizes tumors to checkpoint blockade therapy. Immunity 44:343-354

3. Qiao J, Liu Z, Fu YX (2016) Adapting conventional cancer treatment forimmunotherapy. J Mol Med. doi:10.1007/s00109-016-1393-4

4. Zitvogel L, Ayyoub M, Routy B, Kroemer G (2016) Microbiome and anticancer immunosurveillance. Cell 165:276-287

5. Vétizou M, Pitt JM, Daillère R, Lepage P, Waldschmitt N, Flament C, Rusakiewicz S, Routy B, Roberti MP, Duong CP et al (2015) Anticancer immunotherapy by CTLA-4 blockade relies on the gut microbiota. Science 350:1079-1084

6. Ma Y, Yang H, Pitt JM, Kroemer G, Zitvogel L (2016) Therapyinduced microenvironmental changes in cancer. J Mol Med. doi:10. 1007/s00109-016-1401-8

7. Ruffell B, Coussens LM (2015) Macrophages and therapeutic resistance in cancer. Cancer Cell 27:462-472

8. Hou J, Zhou Y, Zheng Y, Fan J, Zhou W, Ng IO, Sun H, Qin L, Qiu S, Lee JM et al (2014) Hepatic RIG-I predicts survival and interferon- $\alpha$ therapeutic response in hepatocellular carcinoma. Cancer Cell 25:49-63

9. Liu Y, Cao X (2016) Immunosuppressive cells in tumor immune escape and metastasis. J Mol Med. doi:10.1007/s00109-015-1376-x

10. Kryczek I, Lin Y, Nagarsheth N, Peng D, Zhao L, Zhao E, Vatan L, Szeliga W, Dou Y, Owens S et al (2014) IL-22(+)CD4(+) T cells promote colorectal cancer stemness via STAT3 transcription factor activation and induction of the methyltransferase DOT1L. Immunity 40:772-784

11. Perusina Lanfranca M, Lin Y, Fang J, Zou W, Frankel T (2016) Biological and pathological activities of interleukin-22. J Mol Med. doi:10.1007/s00109-016-1391-6

12. Lu B, Yang M, Wang Q (2016) Interleukin-33 in tumorigenesis, tumor immune evasion, and cancer immunotherapy. J Mol Med. doi:10.1007/s00109-016-1397-0 